\title{
HAMBURG/SAO SURVEY OF EMISSION-LINE GALAXIES
}

\author{
V. LIPOVETSKY ${ }^{1}$, D. ENGELS ${ }^{2}$, A. UGRYUMOV ${ }^{1}$, U. HOPP ${ }^{3 *}$, \\ G. RICHTER ${ }^{4}$, Y. IZOTOV $^{5}$, A. KNIAZEV ${ }^{1}$ AND C. POPESCU ${ }^{6}$ \\ ${ }^{1}$ Special Astrophysical Observatory, Russia \\ ${ }^{2}$ Hamburger Sternwarte, Hamburg, Germany \\ 3 Universitätssternwarte München, Munich, Germany \\ 4 AIP, Potsdam-Babelsberg, Germany \\ ${ }^{5}$ Main Astronomical Observatory, Goloseevo, Kiev, Ukraina \\ ${ }^{6}$ MPI für Astronomy, Heidelberg, Germany
}

\section{Introduction}

We present first results of the Hamburg/SAO Survey of emission-line galaxies (hereafter HSS, SAO-Special Astrophysical Observatory, Russia) initiated to search for extremely metal-deficient $\left(Z<Z_{\odot} / 10\right)$ galaxies and to create a large sample of Blue Compact Galaxies (BCG). This "Northern BCG Sample," will be assembled by merging the HSS with samples from the Second Byurakan Survey (SBS) (Stepanian et al. 1987) and the Case Low-Dispersion Northern Sky Survey (Pesch et al. 1991).

The strongly metal-deficient galaxies are important for the estimation of primordial helium abundance-a crucial parameter in Standard Big Bang nucleosynthesis theory - for the understanding of basic processes determining the chemical evolution of galaxies, for constraints to models of stellar nucleosynthesis, and for the determination of the initial stellar mass function. During the last three decades only few galaxies of extremely lowmetallicity such as I Zw 18 or SBS $0335-052$ were found, indicating that they are rare. New surveys are therefore necessary to increase the volume sampled significantly to improve the search. One way is to decrease the limiting [OIII] $\lambda \lambda 4959,5007 \AA$ emission line fluxes as it is done by the KISS project (Kniazev et al. 1996). The other way is to increase the sky area surveyed, keeping the sensitivity to the detection of the [OIII] emission

${ }^{1}$ Visiting astronomer Calar Alto Observatory. 


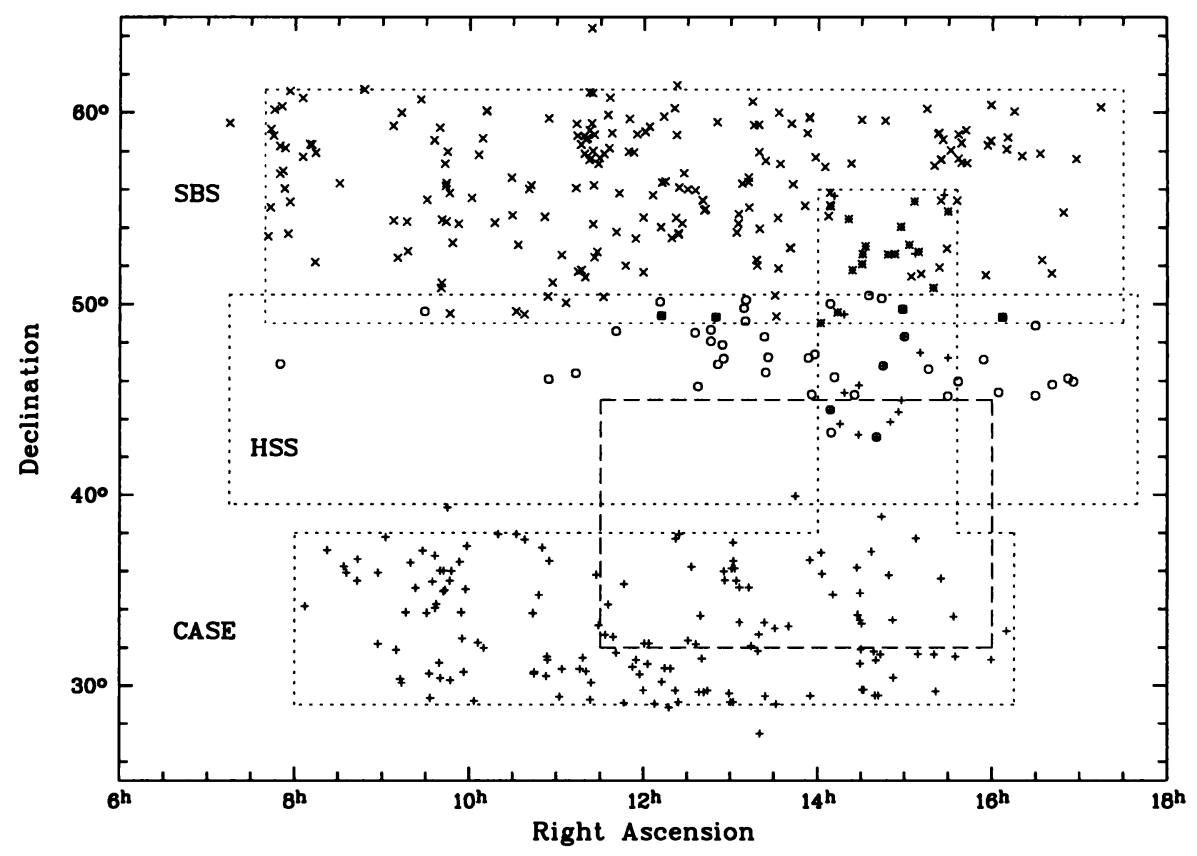

Figure 1. Sky positions of known BCGs from the Northern Sample, drawn from the SBS, HSS and Case surveys.

lines on a level similar to those of previous surveys. Both these strategies should be followed as they are complementary to each other.

BCGs, having strong emission lines in their optical spectra give the opportunity to map the low-mass galaxy population at distances well beyond the Local Supercluster (Salzer 1989; Salzer et al. 1993; Hopp et al. 1995; Pustil'nik et al. 1995). They may help trace the spatial distribution of low-mass galaxies and test cosmological theories of the origin and of the development of large-scale structures in the Universe. However all the most representative samples of BCGs from the SBS, Case and UM surveys are located in narrow bands and yield significant boundary effects for any statistical analysis. The aim of the Hamburg/SAO Survey is not only to provide a new large BCG sample, but also to fill the gap between the areas covered by the SBS and Case surveys.

The three surveys together will constitute the "Northern BCG sample" with a total area on the sky of $\approx 3000 \mathrm{deg}^{2}$. Overlap between the surveys will be used to study selection effects. The current HSS region covers about $1100 \operatorname{deg}^{2}$ within the boundaries: $\alpha=7^{h} 20^{m}-17^{h} 40^{m}$ and $\delta=+40^{\circ}-+50^{\circ}$. We plan to extend it down to $\delta=+35^{\circ}$. 


\section{Selection of Candidates and Follow-Up Spectroscopy}

The selection of candidates of emission-line galaxies was made on digitized objective-prism photoplates of the Hamburg Quasar Survey (HQS; Hagen et al. 1995, see also Popescu et al. 1996 for a related work on emission-line galaxies) during October-December 1994. Spectra with apparent [OIII] emission-lines were selected from the digitized database of low-resolution spectra in semi-automatic mode and then rescanned with full resolution. The high-resolution spectra were classified according to the prominence of the emission lines. Presently, the candidate selection is finished in the region: $\alpha=8^{h}-17^{h} 30^{m}, \delta=45^{\circ}-50^{\circ}$ and $\alpha=8^{h}-15^{h} 30^{m}, \delta=40^{\circ}-45^{\circ}$. First follow-up spectroscopy was obtained in 1995 with the $2.2 \mathrm{~m}$ Calar Alto (German-Spanish Observatory, Spain) and the $6 \mathrm{~m}$ SAO (Russian) telescopes in snap-shot mode. Standard spectral data reduction was performed at the SAO under MIDAS.

\section{Preliminary Results}

Follow-up spectroscopy was performed for 218 candidates, confirming 75 new emission-line objects. 46 of them appear to be BCGs, 4 are QSOs, and the others are different types of galaxies including AGN and starburst. Their magnitude range is $16^{m} \leq B \leq 20^{m}$. About 20 BCGs have low oxygen abundance in the range $12+\log (\mathrm{O} / \mathrm{H})=7.7-8.0$, but no new galaxies with extremely low metallicity were discovered. Comparing with the SBS and Case surveys, we find that the HSS BCG have similar distributions of absolute magnitudes and redshifts. It appears that the galaxies in all three samples have similar physical properties.

\section{References}

Hagen, H.-J., et al. 1995. Astron.Astrophys. Suppl., 11, 195.

Hopp, U., et al. 1995. Astron.Astrophys. Suppl.,109, 537.

Kniazev, A., et al. 1996, this volume, 302

Pesch, P., Sanduleak, N. and Stephenson C.B., 1991. Astrophys.J. Suppl., 76, 1043.

Pustil'nik, S.A., et al. 1995. Astrophys.J., 443, 499.

Popescu, C.C., et al. 1996. Astron.Astrophys. Suppl., 116, 43.

Salzer, J., 1989. Astrophys.J., 347, 152.

Salzer, J. and Rosenberg, J., 1993. In ESO/OHP Workshop on Dwarf galaxies, ed. G. Meylan \& P. Prugniel, p. 129.

Stepanian, J.A., Lipovetsky, V.A., Erastova, L.K. and Shapovalova, A.I., 1987. In "Observational Evidence of Activity of Galaxies," IAU Symp. 128, p. 17. 